\title{
A Sparsity-Based Approach to 3D Binaural Sound Synthesis Using Time-Frequency Array Processing
}

\author{
Maximo Cobos, ${ }^{1}$ Jose J. Lopez (EURASIP Member), ${ }^{1}$ and Sascha Spors (EURASIP Member) ${ }^{2}$ \\ ${ }^{1}$ Institute of Telecommunications and Multimedia Applications, Universidad Politécnica de Valencia, 46022 Valencia, Spain \\ ${ }^{2}$ Deutsche Telekom Laboratories, Technische Universität Berlin, 10578 Berlin, Germany \\ Correspondence should be addressed to Maximo Cobos, mcobos@iteam.upv.es
}

Received 2 March 2010; Revised 21 June 2010; Accepted 7 September 2010

Academic Editor: Augusto Sarti

Copyright ( 2010 Maximo Cobos et al. This is an open access article distributed under the Creative Commons Attribution License, which permits unrestricted use, distribution, and reproduction in any medium, provided the original work is properly cited.

\begin{abstract}
Localization of sounds in physical space plays a very important role in multiple audio-related disciplines, such as music, telecommunications, and audiovisual productions. Binaural recording is the most commonly used method to provide an immersive sound experience by means of headphone reproduction. However, it requires a very specific recording setup using high-fidelity microphones mounted in a dummy head. In this paper, we present a novel processing framework for binaural sound recording and reproduction that avoids the use of dummy heads, which is specially suitable for immersive teleconferencing applications. The method is based on a time-frequency analysis of the spatial properties of the sound picked up by a simple tetrahedral microphone array, assuming source sparseness. The experiments carried out using simulations and a real-time prototype confirm the validity of the proposed approach.
\end{abstract}

\section{Introduction}

Human hearing plays a major role in the way our environment is perceived. Generally, sound is perceived in all three dimensions, width, height, and depth, which are all necessary to achieve a natural perception of sound [1]. These attributes are usually employed to describe the spatial characteristics of sound taking into account its diffuseness properties. The human auditory system is very sophisticated and, thus, capable to analyze and extract most spatial information pertaining to a sound source using two ears. In fact, when a sound scene is recorded by a single microphone, we are still able to recognize the original sound events. However, much of the information corresponding to the spatial properties of these events is lost. As a result, spatial sound recording and reproduction techniques are always based on a multichannel approach.

Reproduction using two-channels or stereo is the most common way that most people know to convey some spatial content into sound recording and reproduction, and this can be considered as the simplest approximation to spatial sound. On the other hand, surround sound systems have evolved and entered homes in order to give a better sensation than stereo by using more reproduction channels and have been widely utilized in theaters since the middle 70s. Both stereo and surround systems have an optimal listening position, known as sweet spot [2]. This optimum listening area is almost limited to the central point in the loudspeaker setup. Outside the central zone, the perceived virtual source locations differ significantly from their intended spatial position.

Another much more realistic strategy is to reproduce directly in the ears of the listener, via headphones, the signal that he/she would perceive in the acoustic environment that is intended to be simulated. This strategy is widely known as binaural reproduction. The signals to be reproduced with headphones can be recorded with an acoustic dummy head or they can be artificially synthesized by using a measured Head-Related Transfer-Function (HRTF) [3]. In an anechoic environment, as sound propagates from the source to the listener, its own head, pinna, and torso introduce changes to the sound before it reaches the ear drums. These effects of the listener's body are registered by the HRTF, which is the transfer function between the sound pressure that is present at the center of the listener's head when the listener is absent 


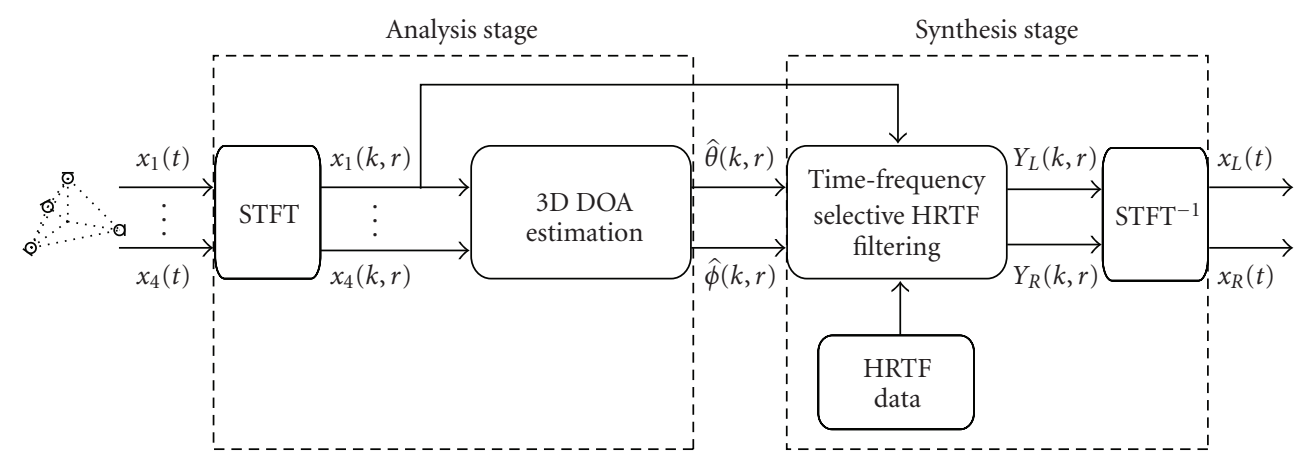

FIGURE 1: Block diagram of the proposed 3D binaural synthesis method.

and the sound pressure developed at the listener's ear. Since humans have different-sized heads, torsos, and ear shapes, HRTFs vary from person to person. The HRTF is a function of direction, distance, and frequency. The inverse Fourier transform of the HRTF is the Head-Related Impulse Response (HRIR), which is a function of direction, distance, and time. Using binaural sound reproduction, it is possible to create a very convincing and immersive sound experience that provides the listener with a natural perception of localized sound events.

In this paper, we present a novel method to capture and process the spatial characteristics of sound with the aim of providing a real-time 3D audio experience. Instead of using an expensive dummy head setup, a small tetrahedral microphone array is utilized to discriminate among the three spatial dimensions, providing a cheap and effective way of constructing a full $3 \mathrm{D}$ audio system. The proposed technique is based on a two-step approach. In a first analysis stage, the signals captured by each microphone pair are processed in the time-frequency domain, resulting in a complete directional description of the recorded sound. In the synthesis stage, source sparseness is assumed, and each time-frequency bin is selectively filtered using a different HRTF depending on its estimated direction.

Figure 1 summarizes the steps involved in the proposed approach for binaural sound synthesis.

(1) The signals obtained by the microphones of the array enter the analysis stage.

(2) In the analysis stage, the four signals are first transformed into the time-frequency domain by means of the Short-Time Fourier Transform (STFT). Then, Direction-of-Arrival (DOA) information (azimuth and elevation) for each time-frequency bin is extracted using the processing described in Section 3.

(3) The synthesis stage is based on a time-frequency selective HRTF filtering of one of the input microphone signals. This filtering is carried out selectively in the STFT domain according to the directions estimated in the analysis stage, resulting in the output signals for the left and right ears. Finally, the ear signals are transformed back to the time domain using the inverse STFT operator.
The paper is structured as follows. Section 2 provides a review of multisource binaural synthesis techniques closely related to our work. Section 3 presents the processing techniques involved in the analysis stage of the method, describing the signal model and the array geometry used to estimate the directional information. Section 4 is devoted to the synthesis stage of the method, where the analyzed spatial information is used to create an immersive 3D sound scene. Section 5 presents a performance comparison between conventional binaural reproduction and our sparsity-based approach using synthetic mixtures of speech and music sources. Section 6 describes and evaluates a real-time prototype that implements the processing described in this paper. Finally, in Section 7, the conclusions of this work are summarized.

\section{Binaural Sound Synthesis}

2.1. Multisource Binaural Sound Synthesis. It is widely known that binaural sound synthesis is a technique capable of reproducing a virtual sound image of a recorded sound signal at an apparent position in the three-dimensional space. Binaural synthesis is based on the use of HRTFs (or their HRIRs time-domain representation) to filter the audio streams corresponding to different sound sources located at different spatial positions, creating a highly immersive audio experience. As a result, to render $N$ sound sources positioned at $N$ different locations it is necessary to use $2 N$ filters $(N$ for the left ear and $N$ for the right ear). The computational complexity is therefore very dependent on the number of sound sources, which makes the real-time rendering of multiple sound sources a very intensive computational task $[4,5]$. In this context, many approaches have been proposed to reduce the complexity of multisource binaural synthesis, many of them based on parametric HRTFs [6]. Experiments with parametric HRTFs have confirmed that subjects cannot discriminate the parametric HRTF versions from the original ones if a suitable set of parameters are selected within each critical band [7]. Breebaart et al. [8] proposed some methods to provide a multichannel audio experience over stereo headphones from a mixdown of sound source signals and a parametric representation (spatial parameters) of the multichannel original signal in a time-frequency domain. The binaural synthesis stage combines the spatial parameters 
of the multichannel signal with the HRTF parameters that describe the virtual loudspeaker setup, resulting in a set of combined binaural parameters that are later used to modify the downmix signal. These rendering methods provide highquality binaural reproduction of multichannel audio and can be easily combined with multichannel audio coders such as MPEG surround.

Despite being powerful and promising, the above approaches are substantially different from the application covered in this paper. The reason is that they are mainly based on a time-frequency analysis of different loudspeaker signals whereas our proposed method takes as input the signals from a small microphone array, which are successfully employed to describe the sound field in one point of the threedimensional space. Therefore, the proposed method shares more similarities with another spatial sound processing technique known as Directional Audio Coding.

\subsection{Directional Audio Coding. Directional Audio Coding} (DirAC) is a recently proposed method for spatial sound recording and reproduction [9] which shares many similarities with the binaural synthesis technique described in this paper. In a first analysis stage, DirAC uses typically a B-format microphone to capture the spatial properties of the sound recorded in a given environment (although other alternatives can also be used [10]). In a second stage, the analyzed spatial features are employed to reproduce the recorded sound again by means of an arbitrary loudspeaker setup. Note that although B-format signals are used, there are substantial differences with conventional Ambisonics reproduction [11].

More recently, a binaural synthesis version of DirAC has been proposed to provide spatial sound reproduction over headphones using the conventional DirAC scheme [12]. The main features of this version and their relation to our proposed approach are next discussed.

2.2.1. DirAC Analysis and Synthesis. The analysis stage of DirAC is based on a time-frequency processing of the Bformat input signals to estimate direction and diffuseness parameters. To this end, an energetic analysis based on pressure and velocity signals is carried out, which needs for an adequate calibration before starting the processing [13]. Besides using a B-format microphone, different array structures can be employed in this analysis stage with the aim of estimating the necessary direction and diffuseness parameters.

Regarding DirAC synthesis, several alternatives have also been proposed. In the low-bit-rate version, only one omnidirectional signal is transmitted along with the spatial metadata, which is used as the signal that is processed and applied to all the reproduction loudspeakers. Another version uses B-format signals to construct a set of virtual microphone signals that are similarly processed using the metadata obtained from the analysis stage [9].

The transmitted signals are divided into two different streams: the diffuse and the nondiffuse sound stream. The nondiffuse sound is assumed to be the part of sound that has a clear direction and is reproduced by the loudspeaker setup using vector base amplitude panning (VBAP) [14]. In contrast, the diffuse sound stream is assumed to surround the listener and the input signal is decorrelated and played from multiple loudspeakers.

The binaural version of DirAC follows a philosophy similar to that of Breebaart's work in that a virtual loudspeaker setup is assumed and implemented by means of HRTF data. Both diffuse and nondiffuse sound streams are processed in the same way as in the real loudspeaker version but using virtual loudspeakers simulated by means of HRTFs [15].

2.2.2. Relation to the Proposed Approach. As previously commented, DirAC shares many similarities with the binaural synthesis method proposed in this paper, which is also based on a two-step approach. However, substantial differences can be found both in the analysis and the synthesis stages of the algorithm.

As will be seen in Section 3.3, amplitude calibration is not necessary in our proposed analysis stage, since DOA estimation is based only on phase information. Although different microphone array alternatives have already been proposed for DOA estimation in a DirAC context, they either are limited to DOA estimation in the horizontal plane [16] or they use more than 4 microphones $[17,18]$. Moreover, as will be later explained, diffuseness is not directly estimated in our proposed approach since the synthesis stage does not rely on this parameter.

On the other hand, the synthesis stage does not assume a virtual loudspeaker setup nor makes a different treatment between diffuse and nondiffuse components. This makes the synthesis processing even more simple than in DirAC. In fact, in our method, diffuseness information is assumed to be inherently encoded by the DOA estimates since the variance found on the directional information over the time-frequency domain is already a representation of the diffuseness characteristics of the recorded sound. In this context, there is no need for assuming a specific loudspeaker reproduction setup since each time-frequency element is binaurally reproduced according to its estimated direction.

\section{Analysis Stage}

3.1. Signal Model. The signals recorded by a microphone array, with sensors denoted with indices $m=1,2, \ldots, M$ in an acoustic environment where $N$ sound sources are present, can be modeled as a finite impulse response convolutive mixture, written as

$$
x_{m}(t)=\sum_{n=1}^{N} \sum_{\ell=0}^{L_{m}-1} h_{m n}(\ell) s_{n}(t-\ell), \quad m=1, \ldots, M,
$$

where $x_{m}(t)$ is the signal recorded at the $m$ th microphone at time sample $t, s_{n}(t)$ is the $n$th source signal, $h_{m n}(t)$ is the impulse response of the acoustic path from source $n$ to sensor $m$, and $L_{m}$ is the maximum length of all impulse responses.

The above model can also be expressed in the STFT domain. This transform divides a time domain signal into a series of small overlapping pieces; each of these pieces is 
windowed and then individually Fourier transformed [19]. Using this transform, the model of (1) can be expressed as

$$
X_{m}(k, r)=\sum_{n=1}^{N} H_{m n}(k) S(k, r) \text {, }
$$

where $X_{m}(k, r)$ denotes the STFT of the $m$ th microphone signal, being $k$ and $r$ the frequency index and time frame index, respectively. $S_{n}(k, r)$ denotes the STFT of the source signal $s_{n}(t)$ and $H_{m n}(k)$ is the frequency response from source $n$ to sensor $m$. Note that (2) is only equivalent to (1) in the case when the analysis window in the computation of the STFT is longer than $L_{m}$.

If we assume that the sources rarely overlap at each timefrequency point, (2) can be simplified as follows:

$$
X_{m}(k, r) \approx H_{m a}(k) S_{a}(k, r),
$$

where $S_{a}(k, r)$ is the dominant source at time-frequency point $(k, r)$. To simplify, we assume an anechoic model where the sources are sufficiently distant to consider plane wavefront incidence. Then, the frequency response is only a function of the time-delay $\tau_{m n}$ between each source and sensor

$$
H_{m n}(k)=e^{\mathrm{j} 2 \pi f_{k} \tau_{m n}}
$$

$f_{k}$ being the frequency corresponding to frequency index $k$.

3.2. Sparsity and Disjointness. Speech and music signals have been shown to be sparse in the time-frequency domain [20]. A sparse source has a peaky probability density function; the signal is close to zero at most time-frequency points, and has large values in rare occasions. This property has been widely applied in many works related to source signal localization $[21,22]$ and separation $[23,24]$ in underdetermined situations, that is, when there are more sources than microphone signals. However, source sparsity alone is useless if the sources overlap to a high degree. The disjointness of a mixture of sources can be defined as the degree of nonoverlapping of the mixed signals. An objective measure of disjointness is the so-called W-Disjoint Orthogonality (WDO) $[25,26]$.

Spectral overlapping depends not only on source sparsity, but also on the mutual relationships between signals. Highly uncorrelated signals will result in a low probability of overlapping. This is even truer for statistically independent signals, since independence is a stronger requirement than uncorrelation. Speech signals most often mix in a random and uncorrelated manner, such as in the cocktail party paradigm. With music mixtures, the situation is different. Their disjointness will vary strongly according to music type. Tonal music will result in strong overlaps in frequency, while atonal music will be more disjoint in frequency [27].

The disjointness properties of speech and music signals are dependent on the window size parameter, which affects the number of frequency bands in the analysis. In particular, the disjointness of speech signals decreases when the window size is very large as a consequence of the reduced temporal resolution. For music signals, frequency disjointness plays a

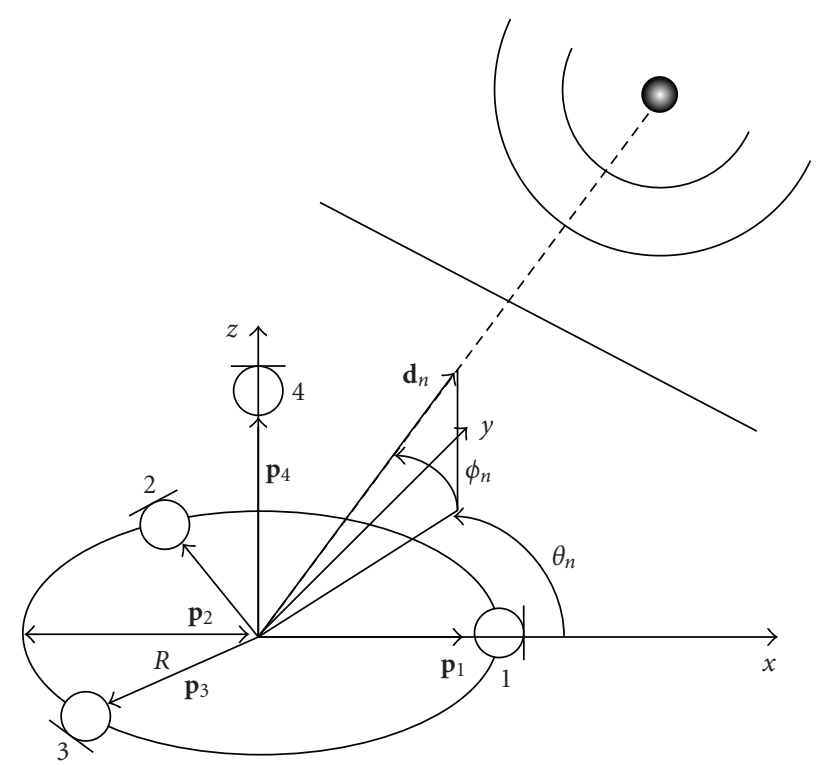

FIGURE 2: Tetrahedral microphone array for 3D DOA estimation.

more important role than time disjointness and so frequency resolution should be favored with longer analysis windows. Moreover, as expected, mixtures of correlated melodies shown to be less disjoint than uncorrelated ones due to the higher amount of spectral and temporal overlapping.

It is also worth to remark that the sparsity and disjointness properties of audio signals become affected in reverberant environments. The room impulse response smears the energy in both time and frequency and so the spectral overlap between different sources in the timefrequency domain is increased with reverberation. Despite this effect, the assumption of nonoverlapping sources has been shown to be still useful for sparsity-based applications such as source separation $[28,29]$.

3.3. Array Geometry and DOA Estimation. Now consider a tetrahedral microphone array $(M=4)$ with base radius $R$, as shown in Figure 2. The sensor location vectors in the 3dimensional space with origin in the array base center, are given by

$$
\begin{aligned}
& \mathbf{p}_{1}=\left[\begin{array}{lll}
R, & 0,0
\end{array}\right]^{T}, \\
& \mathbf{p}_{2}=\left[-\frac{R}{2}, \frac{\sqrt{3}}{2} R, 0\right]^{T}, \\
& \mathbf{p}_{3}=\left[-\frac{R}{2},-\frac{\sqrt{3}}{2} R, 0\right]^{T}, \\
& \mathbf{p}_{4}=\left[\begin{array}{lll}
0, & 0, & R \sqrt{2}
\end{array}\right]^{T} .
\end{aligned}
$$

The DOA vector of the $n$th source as a function of the azimuth $\theta_{n}$ and elevation $\phi_{n}$ angles is defined as

$$
\mathbf{d}_{n}=\left[\cos \theta_{n} \cos \phi_{n}, \sin \theta_{n} \cos \phi_{n}, \sin \phi_{n}\right]^{T} .
$$


The source to sensor time delay with respect to the origin is given by $\tau_{m n}=\mathbf{p}_{m}^{T} \mathbf{d}_{n} / c, c$ being the speed of sound. Therefore, the frequency response of (4) can be written as

$$
H_{m n}(k, r) \approx e^{\mathrm{j}\left(2 \pi f_{k} / c\right) \mathbf{p}_{m}^{T} \mathbf{d}_{n}}
$$

Taking into account this last result and (3), it becomes clear that the phase difference between the microphone pair formed by sensors $i$ and $j$, is given by

$$
\angle\left(\frac{X_{j}(k, r)}{X_{i}(k, r)}\right) \approx \frac{2 \pi f_{k}}{c}\left(\mathbf{p}_{j}-\mathbf{p}_{i}\right)^{T} \mathbf{d}_{n}
$$

where $\angle$ denotes the phase of a complex number.

Using a reference microphone $q$, the phase difference information at point $(k, r)$ of $M-1$ microphone pairs is stored in the vector

$$
\mathbf{b}_{q}(k, r)=\left[\angle\left(\frac{X_{1}(k, r)}{X_{q}(k, r)}\right), \ldots, \angle\left(\frac{X_{M}(k, r)}{X_{q}(k, r)}\right)\right]^{T},
$$

forming the following system of equations:

$$
\mathbf{b}_{q}(k, r)=\frac{2 \pi f_{k}}{c} \mathbf{P d}_{n},
$$

where

$$
\mathbf{P}=\left[\mathbf{p}_{1 q}, \ldots, \mathbf{p}_{M q}\right]^{T}, \quad \mathbf{p}_{n q}=\mathbf{p}_{n}-\mathbf{p}_{q} .
$$

Finally, the DOA at time-frequency bin $(k, r)$ is obtained by taking the inverse of the $\mathbf{P}$ matrix

$$
\widehat{\mathbf{d}}_{n}(k, r)=\frac{c}{2 \pi f_{k}} \mathbf{P}^{-1} \mathbf{b}_{q}(k, r)
$$

The regular tetrahedral geometry used in this paper leads to the following simple equations for $\mathbf{d}_{n}(k, r)=$ $\left[\hat{d}_{1}, \hat{d}_{2}, \hat{d}_{3}\right]^{T}$ :

$$
\begin{aligned}
& \hat{d}_{1}=\cos \theta_{n} \cos \phi_{n}=\frac{c}{2 \pi f_{k}} \frac{1}{\sqrt{3}}\left(b_{2}+b_{3}\right), \\
& \hat{d}_{2}=\sin \theta_{n} \cos \phi_{n}=\frac{c}{2 \pi f_{k}}\left(b_{3}-b_{2}\right), \\
& \hat{d}_{3}=\sin \phi_{n}=\frac{c}{2 \pi f_{k}}\left[\frac{1}{\sqrt{6}}\left(b_{2}+b_{3}\right)-\sqrt{\frac{3}{2}} b_{4}\right],
\end{aligned}
$$

where $b_{n}$ is the $n$th element of the vector $\mathbf{b}_{1}(k, r)$ (reference microphone $q=1$ ). The azimuth angle is obtained using the four quadrant inverse tangent function:

$$
\hat{\theta}_{n}(k, r)=\operatorname{atan}^{360^{\circ}}\left(\hat{d}_{1}, \hat{d}_{2}\right) .
$$

The elevation angle is directly obtained as

$$
\hat{\phi}_{n}(k, r)=\sin ^{-1}\left(\hat{d}_{3}\right) .
$$

Note that for each time-frequency point $(k, r)$, estimating the $3 \mathrm{D}$ direction of arrival is relatively simple, just using the observed phase differences between 3 microphone pairs of the array. Another aspect to consider is spatial aliasing. The distance between microphones determines the angular aliasing frequency. Due to the $2 \pi$ ambiguity in the calculation of the phase differences, the maximum ambiguity-free frequency in a microphone pair subarray would be given by $f_{k}=c / 2 d$, where $d$ is the separation distance between the capsules. Beyond this frequency, there is not a oneto-one relationship between phase difference and spatial direction. However, small arrays with $d \approx 1.5 \mathrm{~cm}$ provide an unambiguous bandwidth greater than $11 \mathrm{kHz}$, covering a perceptually important frequency range.

3.4. Example. With the objective of showing how this analysis stage is capable of capturing the 3D spatial information of sound, we show a simulated sound scene where 4 speech sources are simultaneously active. The simulation has been carried out considering a shoe-box-shaped room $(3.6 \mathrm{~m} \times$ $3.6 \mathrm{~m} \times 2.2 \mathrm{~m}$ ) with reflecting walls (reverberation time $T_{60}=0.1 \mathrm{~s}$ ). The azimuth angles of the sources were $\theta_{1}=15^{\circ}, \theta_{2}=75^{\circ}, \theta_{3}=210^{\circ}$, and $\theta_{4}=260^{\circ}$. The elevation angles were $\phi_{1}=0^{\circ}, \phi_{2}=30^{\circ}, \phi_{3}=-10^{\circ}$, and $\phi_{4}=45^{\circ}$. Figure 3(a) shows the source locations in the 3D space. Figures 3(b) and 3(c) show the 2D histograms of the distribution of DOA estimates in the $\mathrm{XY}$ and $\mathrm{ZY}$ plane, where red color means that many estimates are concentrated on the same location. Note how most DOA estimates are concentrated around the actual source directions. The deviations in the estimates are a consequence of room reflections and interference. The effect of reverberation in sparse source localization was studied by the authors in a previous work [30]. However, as will be explained in the next section, these deviations do not have a negative effect on our proposed binaural synthesis method, since they contribute to the perception of the diffuseness properties of sound.

\section{Synthesis Stage}

As said in Section 1, HRTFs provide accurate localization cues because they encode all the necessary information regarding how the arriving sound is filtered by the diffraction and reflection properties of the head, pinna, and torso, before it reaches the eardrum and inner ear. Using this information, synthesizing a binaural sound signal for headphone reproduction is straightforward. The HRTF for each ear must be used to filter an input signal, ensuring that the outputs are correctly reproduced over their corresponding headphone channel. This is usually done for each separate source signal with the aim of positioning an auditory object in the direction from which the HRTFs have been measured. However, in our proposed approach, the synthesis stage differs significantly from this conventional processing due to the fact that no separate source signals are available. Again, taking into account source sparseness in the time-frequency domain, we will be able to reproduce the original spatial characteristics of the recorded sound scene using the directional information extracted from the previous analysis stage. 


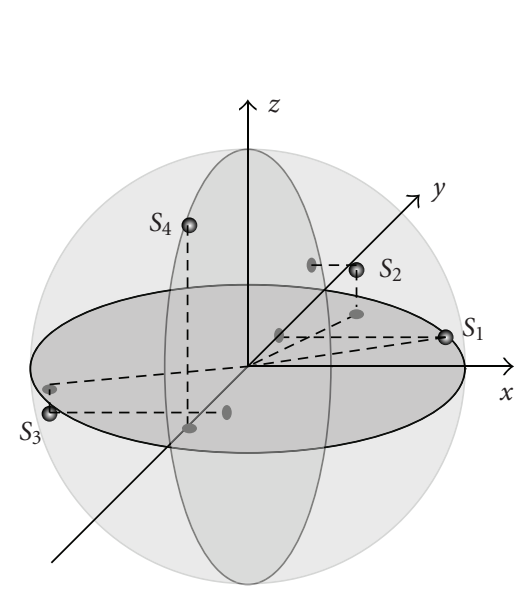

(a)

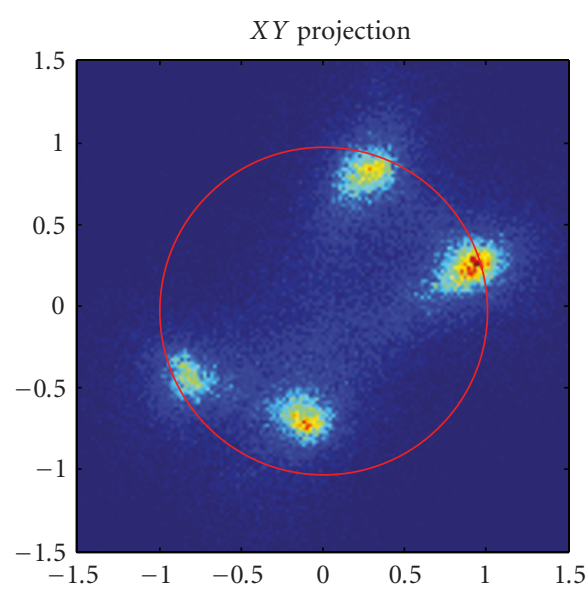

(b)

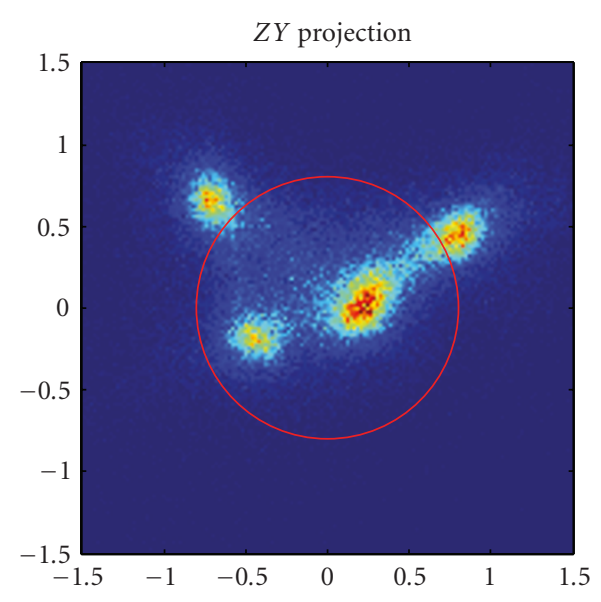

(c)

FIGURE 3: DOA analysis of a mixture of 4 speech sources. (a) Source locations in the 3D space. (b) Distribution of DOA estimates in the XY plane. (c) Distribution of DOA estimates in the ZY plane.

4.1. Time-Frequency Selective HRTF Filtering. Consider a set of measured [31,32] or simulated HRTFs [33]. It is widely known that the use of nonindividualized HRTFs for binaural reproduction has some problems, mainly

(i) sound objects are frequently localized inside the head,

(ii) frontal sounds often appear behind the listener and vice versa,

(iii) the perceived directions of the synthesized sources do not match the intended spatial positions.

These classical problems associated to binaural reproduction have already been extensively studied [34] and we will not address them in this paper.

Assuming far field conditions, the HRTF is a function of the arrival direction of the source $\left(\theta_{n}, \phi_{n}\right)$ and the frequency $f_{k}$, expressed as $\operatorname{HRTF}\left(\theta_{n}, \phi_{n}, k\right)$. Moreover, there is also a different HRTF for the right and left ears, having $\operatorname{HRTF}_{L}\left(\theta_{n}, \phi_{n}, k\right)$ and $\operatorname{HRTF}_{R}\left(\theta_{n}, \phi_{n}, k\right)$.

The synthesis strategy is simple. Any of the omnidirectional signals of the array $X_{m}(k, r)$ is filtered accordingly to the estimated DOA angles $\hat{\theta}_{n}$ and $\hat{\phi}_{n}$ as follows:

$$
\begin{aligned}
& Y_{L}(k, r)=X_{m}(k, r) \operatorname{HRTF}_{L}\left(\hat{\theta}_{n}, \hat{\phi}_{n}, k\right), \\
& Y_{R}(k, r)=X_{m}(k, r) \operatorname{HRTF}_{R}\left(\hat{\theta}_{n}, \hat{\phi}_{n}, k\right),
\end{aligned}
$$

where $Y_{L}(k, r)$ and $Y_{R}(k, r)$ are the STFT of the output signals corresponding to the left and right ears, respectively. These signals are transformed back to the time domain using the inverse STFT operator following an overlap-add scheme.

Using the above approach, the microphone signal $X_{m}(k, r)$ provides a pressure signal for the synthesis of the binaural signal. The required spatial localization cues are then given by the HRTF coefficients, which are carefully selected based on the estimated directional data. Note that we only use a single omnidirectional signal for the calculation of the output, since combinations of the microphone signals
TABLE 1: Mean square error for synthesized signals in anechoic scenario.

\begin{tabular}{lc}
\hline Number of sources $N$ & Mean square error \\
\hline 1 & 0.003 \\
2 & 0.073 \\
3 & 0.243 \\
4 & 0.382 \\
\hline
\end{tabular}

could result in coloration due to spatial filtering effects. In our implementation, we chose the signal of microphone 4 for being slightly above from the array center.

4.2. Selective Filtering and Sparsity. Further considerations are needed regarding the above synthesis approach. Note that each time-frequency bin is independently filtered according to its DOA information. As well as in the analysis stage, source sparsity and disjointness form the basis of our synthesis method. Under approximate WDO conditions, only one source has a major contribution on a given time-frequency element. Therefore, it is only necessary to filter each bin according to the direction of the dominant contribution since the energy corresponding to the rest of the sources can be neglected with little error. Obviously, if the number of sources is increased, the error will be higher.

To illustrate this idea, Figure 4 shows the waveforms of the left and right ear signals in an anechoic scenario for an increasing number of sources. The real signals obtained by conventional HRTF synthesis are shown on the left side and the ones synthesized by means of time-frequency selective filtering are on the right side. To evaluate quantitatively the synthesized signals, their mean square errors are provided in Table 1. As expected, the error of the synthesized signal depends on the number of sources. However, as will be shown in the next section, these errors do not severely affect the subjective quality of the synthesized signals. 

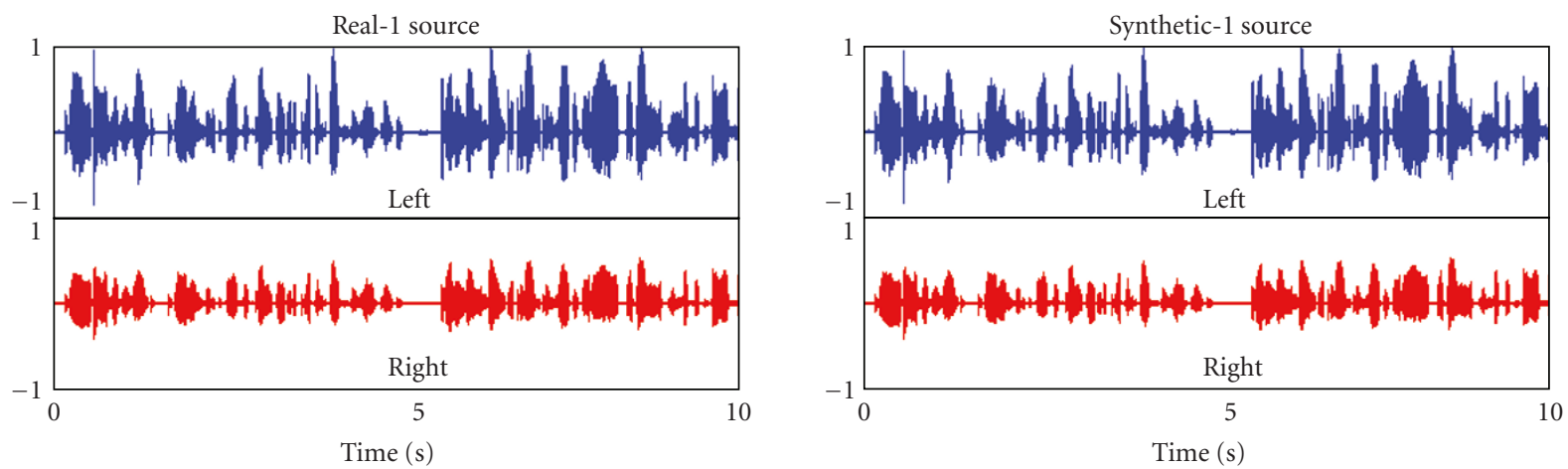

(a)
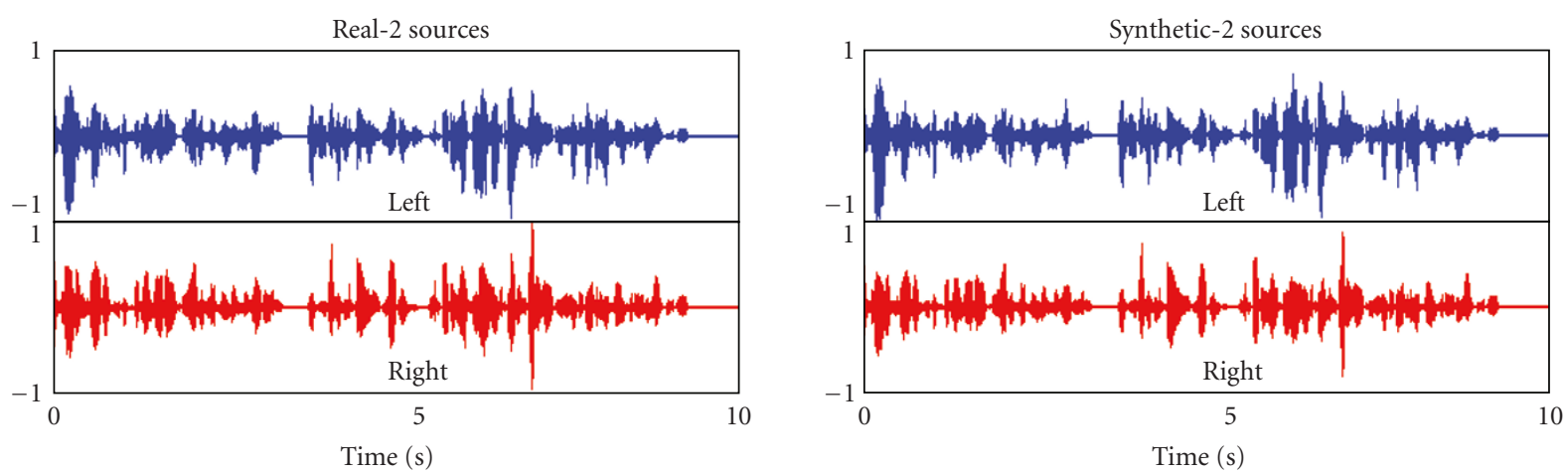

(b)
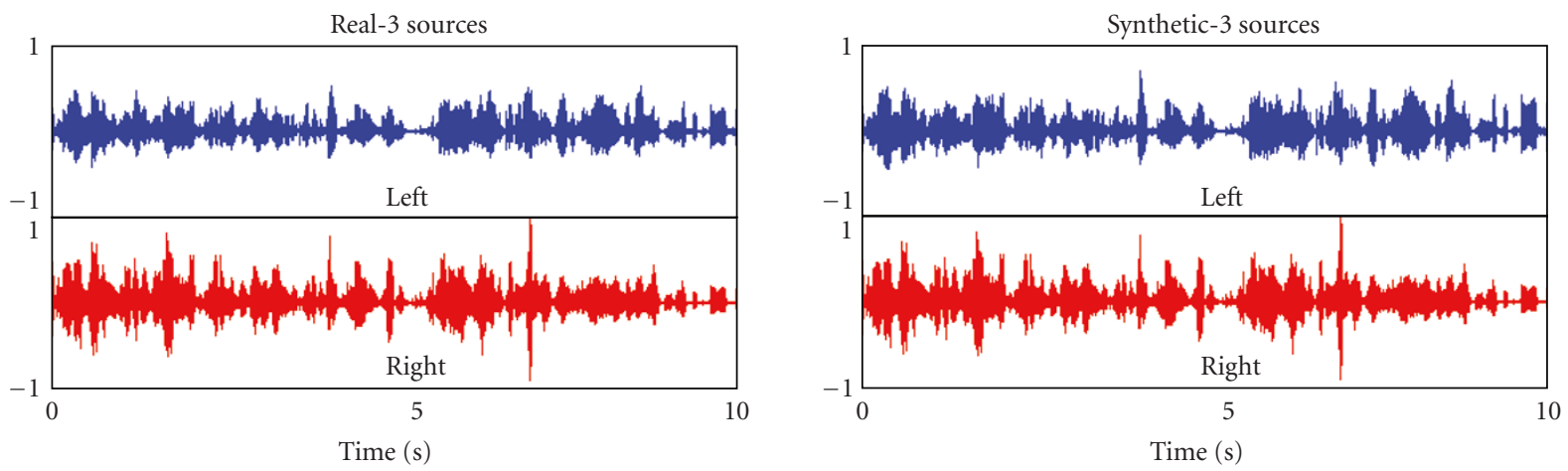

(c)

FIGURE 4: Waveforms of real and sparsity-based synthesized binaural signals for different number of sources. (a) One source. (b) Two sources. (c) Three sources.

Besides the number of sources, the environment and the type of signals also play a major role in our synthesis method. As explained in Section 3.2, speech and music have different sparsity properties and room reflections spread the energy of the sources both in time and frequency. However this is not a serious problem. Time-frequency points dominated by reverberation will be inherently reproduced from multiple directions, just as suggested by the analysis stage. This way, the higher the variance found in the estimated directions, the higher the sense of envelopment will be perceived. In contrast, a dry room that produces very peaky DOA distributions will result in a synthesized binaural signal where the sources can be clearly perceived from their actual directions. A problem associated with a high degree of reverberation is that artifacts may appear due to the prominent discontinuities in the directional data. These effects can be effectively reduced by smoothing the filtering coefficients along the frequency axis.

4.3. HRTF Spatial Resolution. Traditionally, a practical problem associated with HRTFs is the difficulty to measure responses for every possible angle with infinite spatial resolution. Although some approaches have been recently proposed to solve this classical problem [35], most available HRTF databases have been measured with some practical resolution. In order to use HRTFs corresponding to the acquired directional information, several approaches can be followed. 


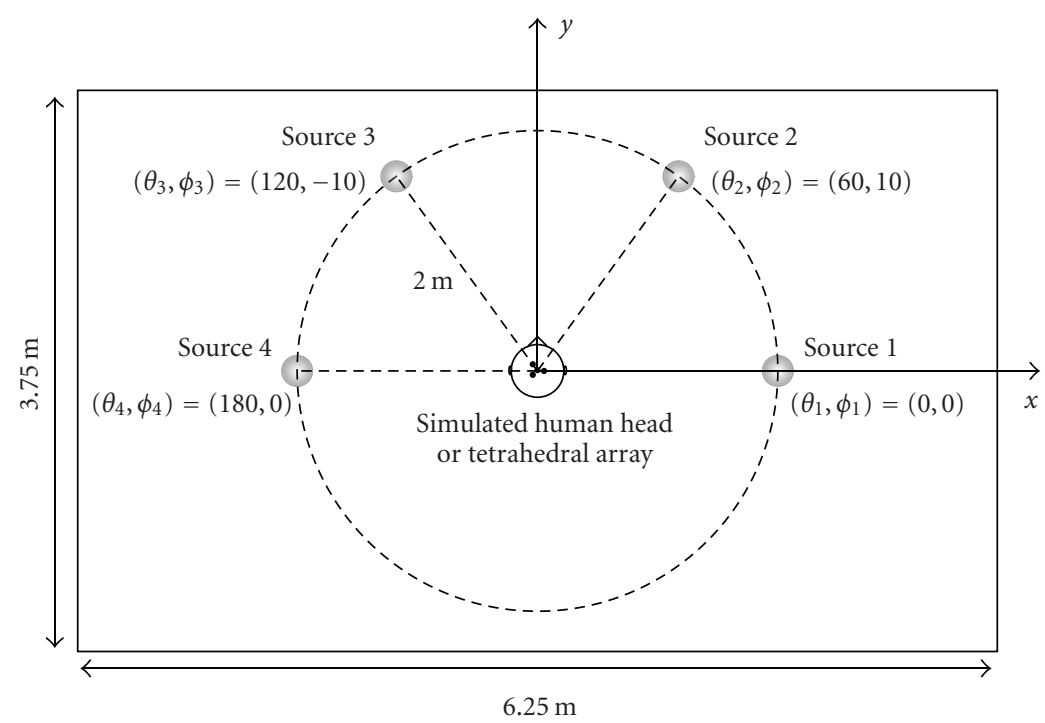

Figure 5: Simulation setup used to obtain the signals used in the subjective evaluation.

(1) Use directly the HRTF of the available data bank that is closest to the estimated direction. This would be the simplest approach.

(2) Interpolate the available HRTFs to get more accurate filters. This can be done using sophisticated interpolation techniques [36-38]. However, a simple linear interpolation in the time domain using the closest HRIRs has shown to be a very convincing and efficient solution $[39,40]$.

(3) Use a parametric HRTF model [33, 41]. This option provides directly the filtering information needed for any direction.

Depending on the requirements of a given application, a different strategy can be selected. While the interpolation strategy is very useful for achieving accurate localization, the other two methods are computationally more efficient. In the next section we comment on some useful aspects regarding the real-time implementation of the method.

\section{Evaluation Using Synthetic Mixtures}

To evaluate subjectively the quality and spatial impression provided by the proposed technique, a set of simulations considering different acoustic situations were carried out. The evaluation conducted this way is useful to assess the performance of the method under different acoustic environments with control on specific aspects of the acoustic setup.

In the experiments, a set of sound sources were simulated inside a shoe-box-shaped room $(6.25 \times 3.75 \times 2.5 \mathrm{~m})$, acquiring all the required impulse responses by means of the Roomsim [42] simulation package for Matlab. This software simulates the acoustics of a rectangular room by means of the image method [43] and, moreover, it allows to generate binaural room impulse responses (BRIRs) corresponding to a selected HRTF data set.
The simulation setup is depicted in Figure 5. Four source positions, were considered in the experiments at a radius $2 \mathrm{~m}$ for the array base center (origin of coordinates): $\left(\theta_{1}=\right.$ $\left.0^{\circ}, \phi_{1}=0^{\circ}\right),\left(\theta_{2}=60^{\circ}, \phi_{2}=10^{\circ}\right),\left(\theta_{3}=120^{\circ}, \phi_{3}=-10^{\circ}\right)$, and $\left(\theta_{4}=180^{\circ}, \phi_{4}=0^{\circ}\right)$. The signals at the microphones were obtained by convolving the simulated responses with the corresponding dry source signals and adding all of them together. To simulate our tetrahedral array, we used an intermicrophone distance of $d=1.5 \mathrm{~cm}$ and assumed perfect omnidirectional responses for all sensors. On the other hand, the KEMAR mannequin [44] was selected to generate reference source signals for the subjective tests.

Different types of signals were considered to take into account different sparsity properties.

(i) A set of 2 male and 2 female speech sources extracted from the public data provided in The 2008 Signal Separation Evaluation Campaign [45]. They are sampled at $16 \mathrm{kHz}, 16$ bits, and have a duration of $10 \mathrm{~s}$.

(ii) A multitrack folk music recording consisting of four instruments: accordion, sax, guitar, and violin. Although originally sampled at $44.1 \mathrm{kHz}$, they were resampled to have the same sampling frequency $(16 \mathrm{kHz})$ as the above speech mixtures.

The STFT was computed using Hann windows of 1024 samples of length, with a hop size of 512 samples $(50 \%$ overlap). These parameters have been shown to be optimum for sparsity-based speech processing [27]. However, music would benefit from longer time windows.

A set of 7 listeners took part on an informal listening test with the aim of evaluating the similarities between the scenes rendered by means of the simulated KEMAR and those obtained by means of the proposed approach. The assessed sound scenes were mixtures of one, two, three, and four sources. There were three versions of each scene. Each version was obtained using different room surface 
characteristics, thus, having different reverberation times: $T_{60}=0 \mathrm{~s}$ (anechoic), $T_{60}=0.1 \mathrm{~s}$ (slightly reverberant), and $T_{60}=0.9$ (very reverberant). As a result, there were 2 versions (KEMAR-simulation and proposed) of a total of 24 different sound scenes (12 for speech and 12 for music).

Two different aspects were considered in the evaluation: sound quality and spatial impression. A 4-point grade scale was used to compare the scenes rendered using the tetrahedral array with the reference KEMAR simulated scenes, ranging from -3 to 0 in the following intensity scale:

(i) 0: Equal,

(ii) -1 : Slightly Worse,

(iii) -2 : Moderately Worse,

(iv) -3 : Substantially Worse.

5.1. Results. Figures 6(a) and 6(b) show the results of the tests for sound quality and spatial impression, respectively. Black dots denote the mean values and thin bars represent $95 \%$ confidence intervals. Regarding sound quality (Figure 6(a)), it can be observed that in anechoic conditions $\left(T_{60}=0\right)$, there are no significant differences between both binaural reproduction methods. However, as the reverberation degree gets higher, the performance of the method is slightly degraded. This worsening may be due to some metallic sound reported by some listeners. There are also clear differences between speech and music, music being considerably more problematic than speech, specially when the number of sound sources is higher. This is a consequence of harmonic overlapping, which affects substantially the WDO assumption. Regarding spatial impression (Figure 6(b)), the decreasing tendency with reverberation is again observed, but the number of sound sources and the type of source signals seem to be less significant.

From the above results, it becomes clear that both source overlapping and reverberation affect negatively the performance of the proposed approach. Obviously, this degradation is due to the fact that some of the assumptions taken for the development of the algorithm are not completely met, specially those based on source sparsity and disjointness. A detailed analysis of the artifacts caused by different types of errors in the analysis and synthesis stages could be useful to improve the performance of the method when working in difficult acoustic environments. Although this analysis is out of the scope of this paper, the authors plan to address this issue in future works.

\section{Evaluation with Real Mixtures}

6.1. Real-Time Implementation. In the last section, a set of experiments using simulations of reverberant rooms were presented. Besides considering these simulations, the applicability of the proposed method can be substantially enhanced by providing some notes on the real-time implementation of a working prototype. Two objectives are pursued with this implementation. First, to demonstrate that the computational cost of this technique is reduced enough to be implemented in a practical embedded system. Second, having a real-time system allowed us to plan future interactive experiments where conditions related to scene changes can be experienced as they occur.

For our real-time prototype we used a PC running Microsoft Windows XP as a base. To construct the microphone array prototype with $d=1.5 \mathrm{~cm}$, four instrumentation quality microphones from Brüel \& Kjaer model 4958 were used. These microphones have excellent phase matching in the audio band. The signal acquiring system consisted of a digital audio interface with four microphone inputs (M-Audio Fast Track Ultra USB 2.0) and ASIO drivers. The Intel Integrated Performance Primitives (Intel IPP) [46] library was used for FFT computation and vector operations. In the analysis stage, phase differences are calculated from the FFT coefficients of each input data frame at each channel. The $[x, y, z]$ components of the DOA vector are then calculated using (13), taking into account that the corresponding frequencies $f_{k}$ have to be previously stored. Moreover, the processing parameters were set the same as in Section 5.

Since the experiments reported in the following subsection were conducted using a Brüel \& Kjaer 4128 Head And Torso Simulator (HATS), the HRTF database used for the synthesis was specifically measured by the authors to allow for an objective comparison. The HRTFs were measured using the logarithmic sweep method [47] with sampling frequency $44.1 \mathrm{kHz}$. Moreover, the measuring system was carefully compensated. HRTFs were sampled both in azimuth and elevation. The dummy-head was placed in a rotating table, measuring responses from $-180^{\circ}$ to $180^{\circ}$ every 5 degrees. On the other hand, elevations were measured from $-40^{\circ}$ to $90^{\circ}$ every 10 degrees. For every measure, the same loudspeaker distance to the center was employed (1 m).

6.2. Evaluation and Discussion. Experiments similar to those presented in Section 5 were carried out using the constructed prototype. Different combinations of sound sources were simultaneously recorded using the tetrahedral microphone array and the HATS, placing the microphone array on top (Figure 7). The sources were reproduced in the horizontal plane over different loudspeakers of our Wave-Field Synthesis array, with azimuth angles of $0,60,120$, and 180 degrees. The room has an approximate reverberation time of $T_{60}=$ $0.2 \mathrm{~s}$. For comparison purposes, the same speech and music signals used in the simulations were selected. The reference signals for the listening test are the simultaneously recorded signals from the artificial head. The same group of subjects took part in the evaluation.

The results of this experiment are shown in Figure 8. As expected, there are many similarities with those in Figure 6 for slight reverberation. Again, results both in sound quality and spatial impression are worse for music signals than for speech signals, specially when the number of sources is high. Moreover, the results confirm that sound quality is more critical than spatial impression, however, the overall score suggests that the perceived quality obtained with the 


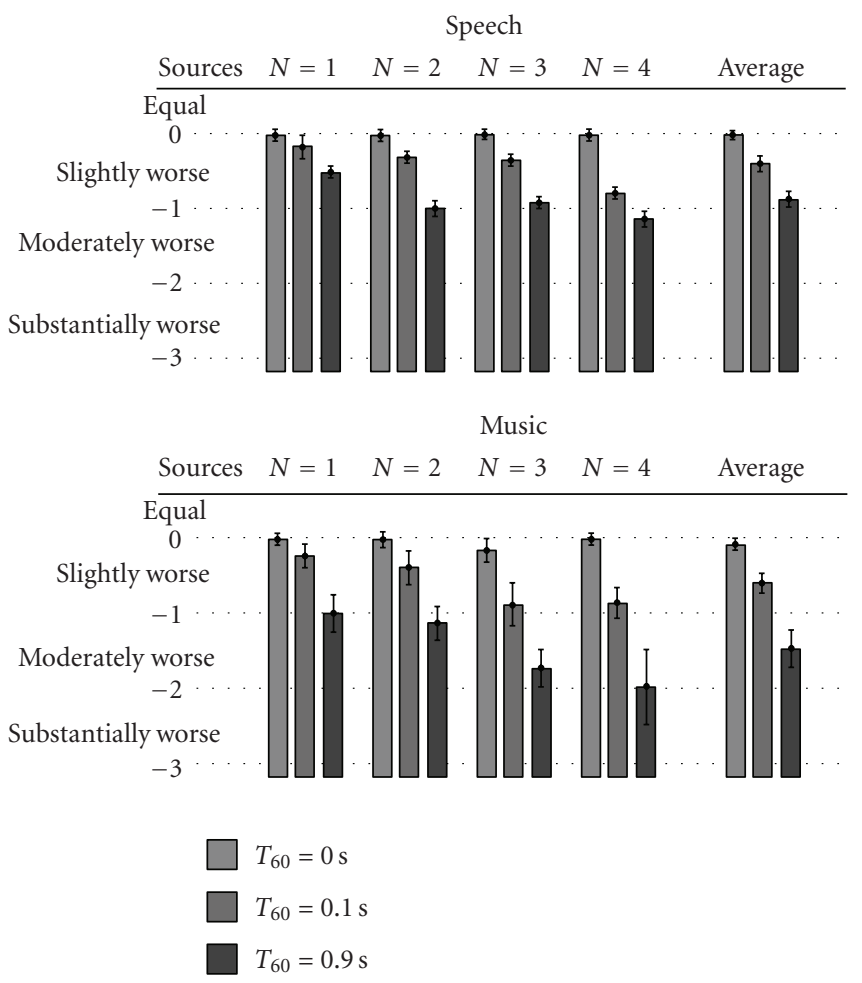

(a)

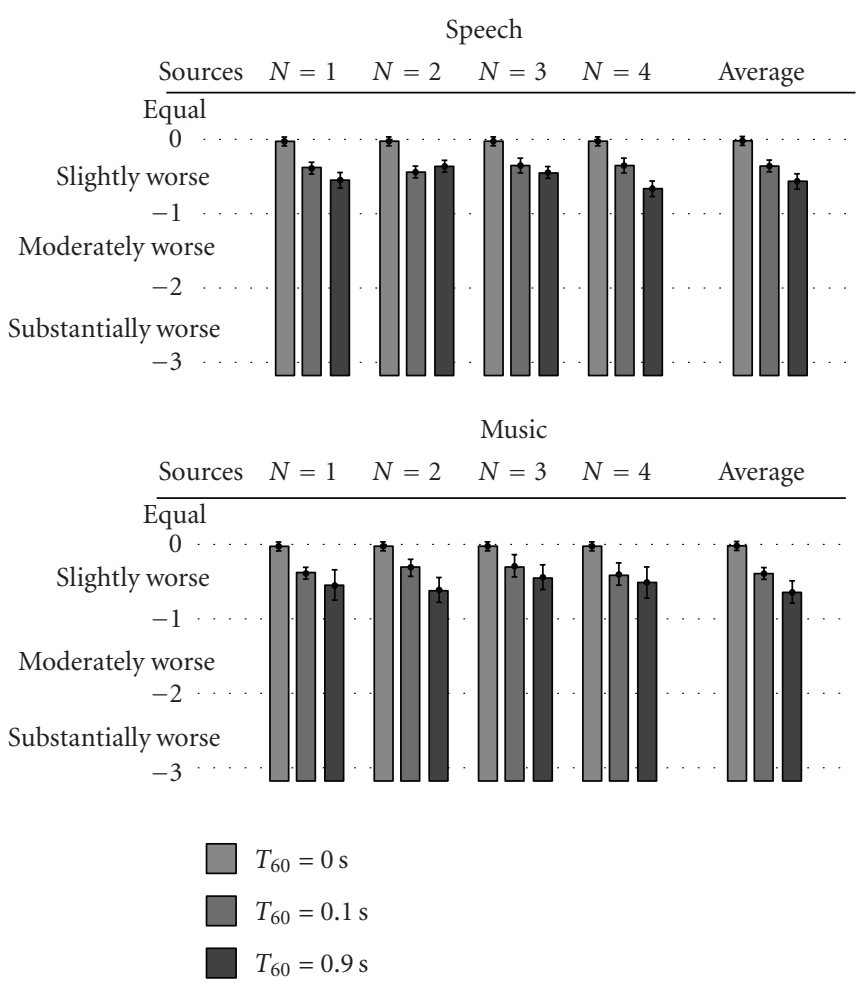

(b)

Figure 6: Results of the subjective tests using synthetic mixtures. Black dots denote the mean values and thin bars represent 95\% confidence intervals. (a) Sound quality evaluation. (b) Spatial impression evaluation.

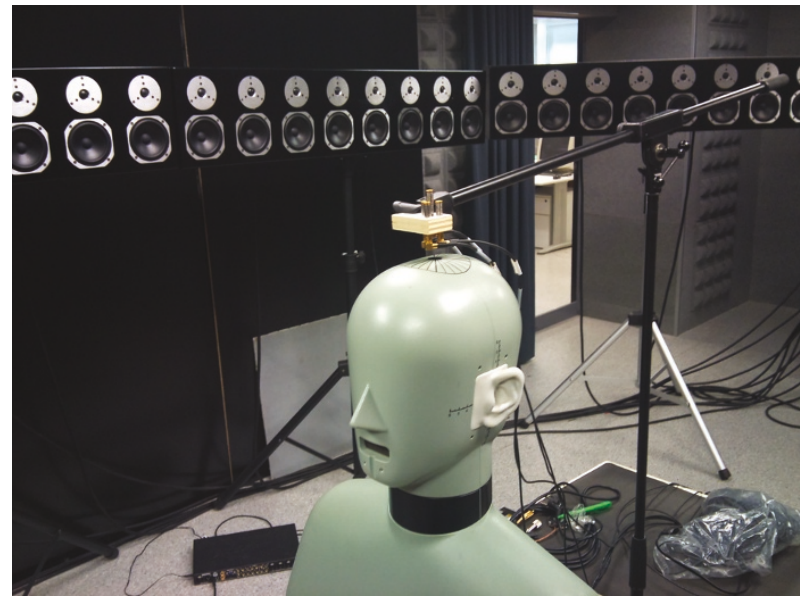

Figure 7: Tetrahedral array and acoustic dummy-head used in the experiments.

proposed synthesis method is only slightly degraded from the obtained using the acoustic dummy-head.

\section{Conclusion}

In this paper, we have presented a two-step binaural sound synthesis method based on sparse signal processing and time-frequency analysis. In the first stage, the assumption of sound sources that rarely overlap in the time-frequency domain has been considered to study the spatial properties of the sound that impinges a small tetrahedral microphone array. The phase difference information of several microphone pairs is combined to obtain a 3D DOA estimate in each time-frequency slot. In the synthesis stage, one of 


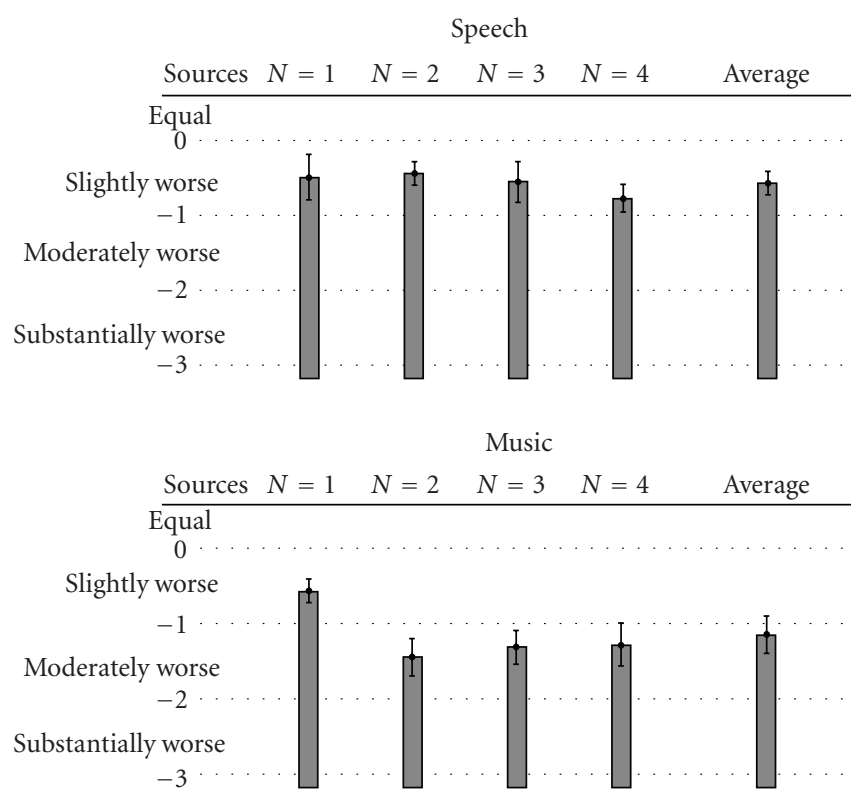

(a)

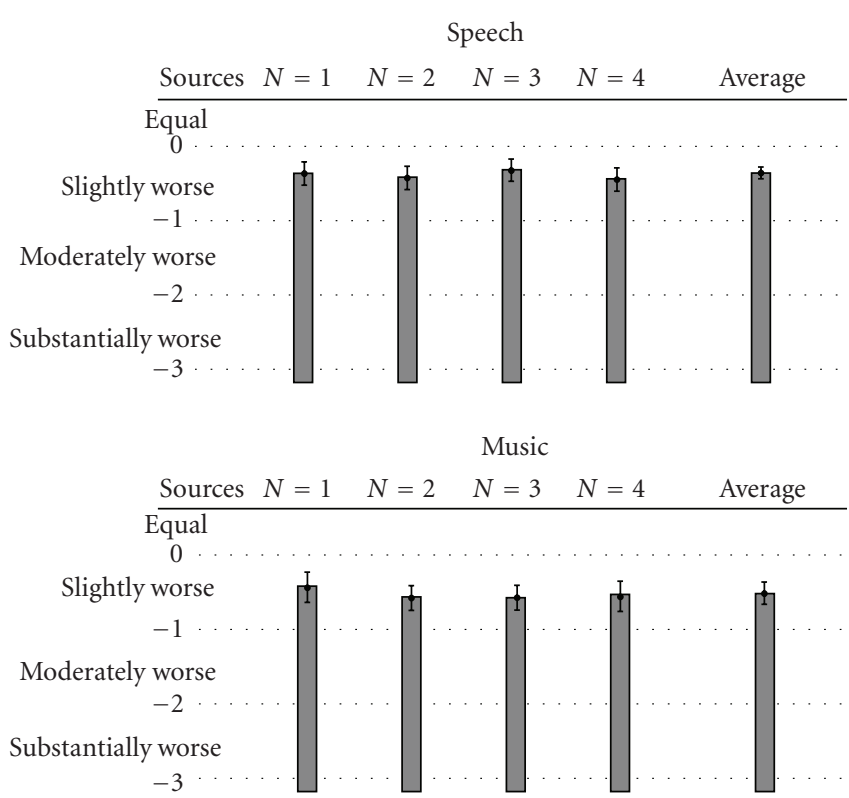

(b)

Figure 8: Results of the subjective tests using real recorded mixtures. Black dots denote the mean values and thin bars represent $95 \%$ confidence intervals. (a) Sound quality evaluation. (b) Spatial impression evaluation.

the microphone signals is selectively filtered in the timefrequency domain with the left and right HRTFs that correspond to the estimated DOAs.

Experiments using both synthetic and real mixtures of speech and music were conducted using different number of sources. Although the performance of the method is slightly degraded with the number of sources and reverberation, the perceived sound quality and spatial impression are considerably similar to conventional binaural reproduction. However, artifacts due to spectral overlapping makes this method more suitable for speech applications than for music.

The proposed spatial sound capturing method not only eliminates the need for an acoustic mannequin, which has a considerable volume and uncomfortable portability, but also allows to change easily the head response by using a different HRTF database in requirement of the application, needs, or user preferences. Moreover, it allows to rotate the head position in real time. Thus, a tracking system can be used to follow the position of the subject in the synthesis stage, providing the listener with a more immersive sensation.

\section{Acknowledgment}

The Spanish Ministry of Science and Innovation supported this work under the Project TEC2009-14414-C03-01.

\section{References}

[1] J. Blauert, Spatial Hearing, MIT Press, Cambridge, UK, 1997.

[2] F. Rumsey, Spatial Audio, Focal Press, 2001.
[3] C. I. Cheng and G. H. Wakefield, "Introduction to headrelated transfer functions (HRTF's): representations of HRTF's in time, frequency, and space," in Proceedings of the 107th Convention of the Audio Engineering Society (AES '99), New York, NY, USA, 1999.

[4] P. S. Chanda, S. Park, and T. I. Kang, "A binaural synthesis with multiple sound sources based on spatial features of headrelated transfer functions," in Proceedings of the International Joint Conference on Neural Networks, pp. 1726-1730, Vacouver, Canada, July 2006.

[5] P. G. Georgiou and C. Kyriakakis, "A multiple input single output model for rendering virtual sound sources in real time," in Proceedings of the IEEE Internatinal Conference on Multimedia and Expo (ICME '00), vol. 1, pp. 253-256, New York, NY, USA, July 2000.

[6] J. Breebaart, F. Nater, and A. Kohlrausch, "Parametric binaural synthesis: background, applications and standards," in Proceedings of the NAG-DAGA, pp. 172-175, Rotterdam, The Netherlands, 2009.

[7] J. Breebaart and C. Faller, Spatial Audio Processing: MPEG Surround and Other Applications, Wiley, Chichester, UK, 2007.

[8] J. Breebaart, L. Villemoes, and K. Kjörling, "Binaural rendering in MPEG surround," EURASIP Journal on Advances in Signal Processing, vol. 2008, Article ID 732895, 14 pages, 2008.

[9] V. Pulkki, "Spatial sound reproduction with directional audio coding," Journal of the Audio Engineering Society, vol. 55, no. 6, pp. 503-516, 2007.

[10] G. Del Galdo and F. Kuech, "Nested microphone array processing for parameter estimation in directional audio coding," in Proceedings of the IEEE Workshop on Applications of Signal Processing to Audio and Acoustics (WASPAA '09), New Paltz, NY, USA, October 2009. 
[11] V. Pulkki, "Directional audio coding in spatial sound reproduction and stereo upmixing," in Proceedings of the AES 28th International Conference, Pitea, Sweden, July 2006.

[12] M. Laitinen and V. Pulkki, "Binaural reproduction for directional audio coding," in Proceedings of the IEEE Workshop on Applications of Signal Processing to Audio and Acoustics (WASPAA '09), pp. 337-340, New Paltz, NY, USA, October 2009.

[13] O. Thiergart, G. Del Galdo, M. Taseska, J. Pineda, and F. Kuech, "In situ microphone array calibration for parameter estimation in directional audio coding," in Proceedings of the AES 128th Convention, London, UK, May 2010.

[14] V. Pulkki, "Spatial sound generation and perception by amplitude panning techniques," Tech. Rep., Helsinki University of Technology, Helsinki, Finland, 2001.

[15] M. Laitinen, Binaural reproduction for directional audio coding, M.S. thesis, Helsinki University of Technology, Helsinki, Finland, 2008.

[16] J. Ahonen, V. Pulkki, and T. Lokki, "Teleconference application and B-format microphone array for directional audio coding," in Proceedings of the AES 30th International Conference, Saariselkä, Finland, March 2007.

[17] R. Schultz-Amling, F. Kuech, M. Kallinger, G. Del Galdo, J. Ahonen, and V. Pulkki, "Planar microphone array processing for the analysis and reproduction of spatial audio using directional audio coding," in Proceedings of the AES 124th Convention, Amsterdam, The Netherlands, May 2008.

[18] J. Merimaa, "Applications of a 3-d microphone array," in Proceedings of the AES 112th Convention, Munich, Germany, May 2002.

[19] L. Cohen, Time-Frequency Analysis, Prentice-Hall, 1995.

[20] J. J. Burred and T. Sikora, "On the use of auditory representations for sparsity-based sound source separation," in Proceedings of the 5th International Conference on Information, Communications and Signal Processing (ICICS '05), pp. 14661470, Bangkok, Thailand, December 2005.

[21] S. Araki, H. Sawada, R. Mukai, and S. Makino, "Performance evaluation of sparse source separation and DOA estimation with observation vector clustering in reverberant environments," in Proceedings of the International Workshop on Acoustic Echo and Noise Control(IWAENC '06), Paris, France, 2006.

[22] S. Rickard and F. Dietrich, "DOA estimation of many wdisjoint orthogonal sources from two mixtures using DUET," in Proceedings of the 10th IEEE Workshop on Statistical Signal and Array Processing (SSAP '00), pp. 311-314, Pocono Manor, $\mathrm{Pa}$, USA, August 2000.

[23] Ö. Yilmaz and S. Rickard, "Blind separation of speech mixtures via time-frequency masking," IEEE Transactions on Signal Processing, vol. 52, no. 7, pp. 1830-1847, 2004.

[24] P. Bofill and M. Zibulevsky, "Underdetermined blind source separation using sparse representations," Signal Processing, vol. 81, no. 11, pp. 2353-2362, 2001.

[25] S. Rickard and O. Yilmaz, "On the w-disjoint orthogonality of speech," in Proceedings of the IEEE International Conference on Acoustics, Speech, and Signal Processing, pp. 529-532, Orlando, Fla, USA, May 2002.

[26] A. Jourjine, S. Richard, and O. Yilmaz, "Blind separation of disjoint orthogonal signals: demixing $\mathrm{n}$ sources from 2 mixtures," in Proceedings of the IEEE International Conference on Acoustics, Speech and Signal Processing (ICASSP '00), vol. 5, pp. 2985-2988, Istanbul, Turkey, 2000.
[27] J. J. Burred, From sparse models to timbre learning: new methods for musical source separation, Ph.D. thesis, Technical University of Berlin, 2008.

[28] S. Schulz and T. Herfet, "On the window-disjoint-orthogonality of speech sources in reverberant humanoid scenarios," in Proceedings of the 11th International Conference on Digital Audio Effects (DAFx '08), Espoo, Finland, September 2008.

[29] M. Cobos and J. J. Lopez, "Two-microphone separation of speech mixtures based on interclass variance maximization," Journal of the Acoustical Society of America, vol. 127, no. 3, pp. 1661-1672, 2010.

[30] M. Cobos, J. J. Lopez, and S. Spors, "Effects of room reverberation in source localization using small microphone arrays," in Proceedings of the 4th International Symposium on Communications, Control and Signal Processing (ISCCSP '10), Limassol, Cyprus, March 2010.

[31] V. R. Algazi, R. O. Duda, D. M. Thompson, and C. Avendano, "The CIPIC HRTF database," in Proceedings of the IEEE Workshop on Applications of Signal Processing to Audio and Acoustics, pp. 99-102, New Paltz, NY, USA, October 2001.

[32] IRCAM, "LISTEN HRTF database," 2003, http://recherche ircam.fr/equipes/salles/listen/.

[33] C. P. Brown and R. O. Duda, "An efficient HRTF model for 3D sound," in Proceedings of the IEEE Workshop on Applications of Signal Processing to Audio and Acoustics (ASSP '97), 1997.

[34] H. Møller, M. F. Sørensen, C. B. Jensen, and D. Hammershøi, "Binaural technique: do we need individual recordings?" Journal of the Audio Engineering Society, vol. 44, no. 6, pp. 451468, 1996.

[35] G. Enzner, "3-d-continuous-azimuth acquisition of headrelated impulse responses using multi-channel adaptive filtering," in Proceedings of the IEEE Workshop on Applications of Signal Processing to Audio and Acoustics (WASPAA '09), pp. 325-328, New Paltz, NY, USA, October 2009.

[36] F. Keyrouz and K. Diepold, "Efficient state-space rational interpolation of HRTFs," in Proceedings of the AES 28th International Conference, Pitea, Sweden, 2006.

[37] F. Freeland, L. Biscainho, and P. Diniz, "Efficient HRTF interpolation in 3D moving sound," in Proceedings of the AES 22nd International Conference on Virtual, Synthetic and Entertainment Audio, Espoo, Finland, June 2002.

[38] F. Keyrouz and K. Diepold, "A new HRTF interpolation approach for fast synthesis of dynamic environmental interaction," Journal of the Audio Engineering Society, vol. 56, no. 1-2, pp. 28-35, 2008.

[39] J. Sodnik, R. Sušnik, M. Štular, and S. Tomažič, "Spatial sound resolution of an interpolated HRIR library," Applied Acoustics, vol. 66, no. 11, pp. 1219-1234, 2005.

[40] T. Nishino, S. Mase, S. Kajita, K. Takeda, and F. Itakura, "Interpolating HRTF for auditory virtual reality," Journal of the AcousticalSociety of America, vol. 100, no. 4, pp. 2602-2602, 1996.

[41] V. Algazi, R. Duda, and D. M. Thomson, "The use of headand-torso models for improved spatial sound synthesis," in Proceedings of the AES 113th Convention, Los Angeles, Calif, USA, October 2002.

[42] D. R. Campbell, "Roomsim: a MATLAB simulation shoebox room acoustics," 2007, http://media.paisley.ac.uk/ campbell/ Roomsim/.

[43] J. B. Allen and D. A. Berkley, "Image method for efficiently simulating small-room acoustics," Journal of the Acoustical Society ofAmerica, vol. 65, no. 4, pp. 943-950, 1979. 
[44] B. Gardner and K. Martin, "HRTF measurements of a KEMAR dummy-head microphone," MIT Media Lab, May 1994, http://alumni.media.mit.edu/ kdm/hrtfdoc/hrtfdoc.html.

[45] E. Vincent, S. Araki, and P. Bofill, "The 2008 signal separation evaluation campaign: a community-based approach to largescale evaluation," in Independent Component Analysis and Signal Separation, vol. 5441 of Lecture Notes in Computer Science, pp. 734-741, 2009.

[46] S. Taylor, Intel Integrated Performance Primitives, Intel Press, 2004.

[47] S. Müller and P. Massarani, "Transfer-function measurement with sweeps," Journal of the Audio Engineering Society, vol. 49, no. 6, pp. 443-471, 2001. 